\title{
DESARROLLO CORPORAL Y TESTICULAR EN CAPRINOS CRIOLLOS SERRANOS DE DIFERENTES ÉPOCAS DE NACIMIENTO
}

\author{
CORPORAL AND TESTICULAR DEVELOPMENT IN MALE CREOLE GOATS \\ AT DIFFERENT BIRTH SEASONS
}

De la Vega, A.C. ${ }^{1 *}$, Arce, O.E. ${ }^{1}$ y Wilde, O.R. ${ }^{1}$

\begin{abstract}
${ }^{1}$ Facultad de Agronomía y Zootecnia. Universidad Nacional de Tucumán. Florentino Ameghino s/n. Bo. Mercantil, El Manantial. CP 4105. Tucumán. Argentina. *adlv@faz.unt.edu.ar

\section{Palabras CLAVE ADICIONALES}

Peso corporal. Circunferencia escrotal. Tucumán.

AdDITIONAL KEYWORDS

Body weight. Scrotal circumference. Tucuman.
\end{abstract}

\section{RESUMEN}

Sobre dos grupos (nacidos en primavera y otoño) de 12 caprinos Criollos Serranos machos, se tomaron durante 28 quincenas consecutivas medidas del peso corporal (PC) y circunferencia escrotal (CE) que resultaron correlacionados $(r=$ $0,82, p<0,001)$. Se registró $(p<0,001)$ interacción tiempo-época para PC con autocorrelación de orden $1(p<0,001)$ entre medidas sucesivas. Se ajustaron ecuaciones marginales de predicción polinómicas de grado 1 para PC y de grado 3 para $\mathrm{CE}$. La época de nacimiento influye tanto en la evolución del PC como de la CE.

\section{SUMMARY}

Two groups (born in spring and autum) of 12 male Criollo Serrano goats were studied for body weight (PC) and scrotal circumference (CE) during 28 consecutive fortnights. PC and CE were correlated $(r=0.82, p<0.001)$. Interaction between time and season was registered $(p<0.001)$ for PC with autocorrelation grade $1(p<0.001)$ between sucessive measures. Predictive marginal equations grade 1 were fitted for $\mathrm{PC}$ and grade 3 for $\mathrm{CE}$. The season of born, influences the evolution of $P C$ and $C E$.

\section{INTRODUCCIÓN}

En majadas Criollas locales se ha observado que los partos se producen tanto en la estación reproductiva tradicional como fuera de ella (Molina et al., 1997; Rabasa et al., 2001). Asimismo se determinó que las varia-

Recibido: 25-5-07. Aceptado: 17-1-08. ciones en el tamaño testicular de caprinos Criollos adultos son ajenas al fotoperiodo (De la Vega et al., 2006). Esto coincide con Pérez Llano y Mateo Rex (1996) respecto a que en latitudes menores a $30^{\circ}$ la estacionalidad es nula o poco marcada y con Alexandre et al. (2001), que califican al Criollo de las Antillas como reproductor continuo. Chemineau (1986) determinó que el fotoperiodo tiene baja incidencia en animales adultos del Caribe, pero que la edad a la primera cubrición depende de la época de nacimiento (Chemineau, 1993).

El objetivo de este trabajo fue determinar la influencia de la época de nacimiento sobre el peso corporal (PC) y circunferencia escrotal (CE) en caprinos Criollos Serranos y la correlación entre ambas variables.

\section{MATERIAL Y MÉTODOS}

El trabajo se realizó en el Campo Experimental de la Facultad de Agronomía y Zootecnia, Universidad Nacional de Tucumán, $\left(26^{\circ} 51^{\prime} \mathrm{S}\right)$. Se diferenciaron dos grupos de animales (nacidos en primavera y otoño) y de cada uno se tomó una muestra aleatoria de 12 individuos. El peso nacimiento promedio fue de 2,95 y $3,35 \mathrm{~kg}$ en otoño y primavera respectivamente. El manejo de los animales durante la etapa de cría fue el mismo, 


\section{DE LA VEGA, ARCE Y WILDE}

aunque las distintas épocas de nacimiento marcan diferencias en las temperaturas que soportan las hembras durante la gestación y en el tipo de pastos al que acceden. Los animales se llevaron al módulo experimental con 4 meses. La alimentación se basó en pastos naturales y aporte de heno de alfalfa durante las horas de encierre, y ración complementaria a base de maíz y harina de soja. La primera medición se consideró tiempo 0 , repitiendo las medidas cada 14 días hasta 18 meses de edad. La CE se midió con escrotímetro de cinta metálica, aplicado en la región ecuatorial del escroto. El PC se determinó con balanza. Al iniciar la experiencia los animales de primavera y otoño tenían 144 y 142 días de vida, 16,8 y 12,2 kg de PC y 20,6 y $17,7 \mathrm{~cm}$ de CE, respectivamente.

Se aplicó la metodología de modelos lineales mixtos de Pinheiro y Bates (2000). Se buscó modelar la curva promedio de respuesta (marginal) a partir de un ajuste individual para cada animal, probando diferentes modelos. Se inició el modelado utilizando polinomios de alto grado y se eliminaron los componentes no significativos. La comparación de modelos alternativos se hizo mediante los criterios de información de Akaike y bayesiano. En el caso de que más de un modelo brindara un adecuado ajuste y no existiendo diferencias significativas entre ellos, se optó por el más sencillo. El grado de ajuste global del modelo final seleccionado se midió mediante el coeficiente $\mathrm{R}^{2}$. Se consideró a los individuos como una muestra aleatoria dentro de cada época. Para evaluar la significación de los parámetros ajustados en cada curva marginal, se utilizaron pruebas $\mathrm{F}$ condicionales.

Tabla I. Ecuaciones marginales para PC. (Marginal equations for the PC).

Nacimiento Ecuación de predicción

Otoño $\quad P C=12,319+0,053$ * tiempo $p<0,001$

Primavera $\mathrm{PC}=17,111+0,037^{*}$ tiempo $\mathrm{p}<0,001$

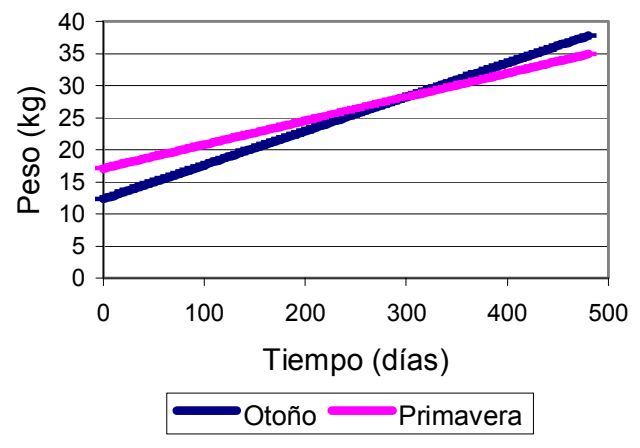

Figura 1. Evoluciónde los pesos según modelo predictivo. (Weight evolution according to predictive model).

Para modelar la falta de independencia entre observaciones repetidas dentro de cada animal, se usó correlación autorregresiva. Las estimaciones de los parámetros se obtuvieron mediante máxima verosimilitud restringida (REML) utilizando el paquete $n$ lme incluido en R (R Development Core Team, 2006). La correlación entre variables fue calculada utilizando el paquete InfoStat (2004).

\section{RESULTADOSYDISCUSIÓN}

Para PC se definió un modelo lineal mixto que incluye el tiempo y el animal como factores aleatorios; los factores fijos fueron el peso, la época y el tiempo $\left(\mathrm{R}^{2}=0,92\right)$. El factor tiempo se consideró fijo para modelar la curva promedio y aleatorio para diferenciar la tendencia individual de cada animal. Se determinó una autocorrelación de orden 1 , cada medición tiene una alta correlación $(\mathrm{r}=0,99)$ con la medición inmediata posterior. Se encontró una interacción significativa $(\mathrm{p}<0,001)$ entre tiempo y época, cada animal se acomoda alrededor de la curva promedio correspondiente con una varianza que le es propia. Las ecuaciones marginales de predicción (polinómicas de grado 1) se exponen en la tabla I; se comenzó probando polinomios de mayor grado pero los de grado 3 y 4 no resultaron significativos. La

Archivos de zootecnia vol. 59, núm. 226, p. 292. 


\section{DESARROLLO CORPORAL Y TESTICULAR EN CAPRINOS CRIOLLOS SERRANOS}

figura 1 presenta las curvas marginales (promedio) modeladas que responden a las ecuaciones de predicción.

Para CE se definió un modelo que únicamente incluye el animal como efecto aleatorio; los factores fijos fueron la $\mathrm{CE}$, la época y el tiempo (lineal, cuadrático y cúbico). También se ajustó una autocorrelación regresiva de orden $1(\mathrm{r}=0,98)$. En este caso, el ajuste de un polinomio de grado 3 con coeficientes diferentes para cada época resultó el más apropiado para la variación temporal de la circunferencia escrotal $\left(\mathrm{R}^{2}=0,86\right)$. Las ecuaciones de predicción marginales se exponen en la tabla II. En este caso, el componente tiempo lineal de la ecuación de primavera no es significativo, pero al resultar altamente significativos los componentes cuadrático y cúbico, el lineal no puede ser ignorado. La figura 2 presenta las curvas marginales modeladas.

En el conjunto de los animales la correlación es alta y positiva entre PC y CE ( $\mathrm{r}=$ 0,82 ). Si se discriminan por época de nacimiento, se obtiene 0,88 en otoño y 0,74 en primavera $(\mathrm{p}<0,001)$.

En la franja tropical y subtropical se ha reportado un período de actividad sexual casi continuo en los caprinos (Molina et al., 1997; Rabasa et al., 2001; Alexandre et al., 2001). El macho adulto originario de zonas tropicales no presenta estacionalidad y puede reproducirse todo el año (Chemineau, 1986) aunque el Criollo de Guadalupe, que no es estacional de adulto, manifiesta marcadas variaciones en la entrada a la pubertad según época de nacimiento (Chemineau, 1993). De la Vega et al. (2002) determinaron que en Criollo Serrano existe influencia de la época de nacimiento sobre el desarrollo testicular en la primera etapa de vida, lo que

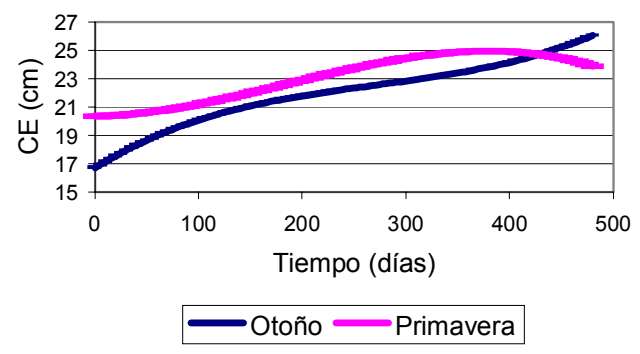

Figura 2. Evolución marginal de la circunferencia escrotal según modelo predictivo. (Marginal SC evolution according to predictive model).

coincide con lo observado aquí en la CE. Los nacidos en primavera presentaron un desarrollo testicular más importante al inicio de las mediciones (fotoperiodo decreciente). La CE de los nacidos en otoño fue inicialmente más baja, alcanzando valores similares a los 18 meses de edad.

Las diferencias entre camadas no pueden atribuirse sólo al fotoperiodo, ya que las épocas de nacimiento, en un sistema pastoril subtropical, influyen sobre la nutrición como han señalado Charallah et al. (2000) y Vera et al. (2002a) en caprinos de zonas tropicales o subtropicales y también De la Vega et al. (2006) en Criollo Serrano adulto.

El peso registra un comportamiento similar al de la CE. Los animales de primavera presentaron mayor peso inicial, pero la pendiente de la curva es más suave. Los nacidos en otoño tienen una mayor velocidad de crecimiento a partir de los 4 meses, lo que podría estar influenciado por los menores requerimientos de mantenimiento de los animales más livianos. Las fluctuaciones de peso a lo largo de la etapa de desarrollo no son suficientes como para ser detectadas

Tabla II. Ecuaciones de predicción marginales definidas para CE en otoño y primavera. (Marginal equations of prediction defined for the CE in autum and spring).

\begin{tabular}{llr}
\hline Otoño & $C E=16,666+0,045^{*} T-0,00013^{*} T^{2}+0,00000016 * T^{3 * * *}$ & $p<0,001$ \\
Primavera & $C E=20,332+0,001 * T+0,00009 * T^{2}-0,00000016 * T^{3 * * *}$ & $p<0,001$
\end{tabular}




\section{DE LA VEGA, ARCE Y WILDE}

por el modelado, por lo que el modelo más sencillo de mejor ajuste corresponde a un polinomio de grado 1 .

La alta correlación entre PC y CE señalan la importancia del estado corporal para un adecuado desarrollo testicular de acuerdo con lo determinado por Souza et al. (2000)

\section{BIBLIOGRAFÍA}

Alexandre, G., Matheron, G., Chemineau, P., Fleury, J. and Xandé, A. 2001. Reproductive performance of Creole goats in Guadalupe (French West Indies) 1. Station-based data. Livest. Res. Rural Dev., 13: 3. http://www.cipav.co//rrd13/ 3/alex133.htm. 12 pp. (19/11/06).

Charallah, S., Khammar, F., Amirat, Z. and Lakhdari, Y. 2000. Sexual activity of Bedouin goat (Capra hircus): zootechnical and nutritional characterisation. In: $7^{\text {th }}$ International conference on goats. France. $460 \mathrm{pp}$.

Chemineau, P. 1986. Sexual behaviour and gonadal activity during the year in the tropical Creole meat goat. II Male mating behaviour, testis diameter ejaculate characteristics and fertility. Reprod. Nutr. Develop., 26: 453-460.

Chemineau, P. 1993. Reproducción de las cabras originarias de las zonas tropicales. Rev. Latamer. Peq. Rumiantes, 1: 2-13.

De La Vega, A.C, Hernández, M.E., Zimerman, M., Vitriu, S. y Ruiz, R. 2002. Influencia de dos épocas de nacimiento en el desarrollo escrotal de caprinos Criollos de origen serrano. XIX Jornadas Científicas de la Sociedad de Biología de Tucumán. Taif del Valle, Tucumán. Argentina. $271 \mathrm{pp}$.

De La Vega, A.C., Morales, P., Zimerman, M. y Wilde, O. 2006. Variación anual de la circunferencia escrotal en caprinos criollos serranos. Arch. Zootec., 55: 113-116.

InfoStat. 2004. InfoStat. Versión 2004. Grupo InfoStat, FCA. Universidad Nacional de Córdoba. Argentina.

Molina, S., Fernández, J., Fernández, M. y Martín, G. (h). 1997. Frecuencia y distribución mensual de pariciones en majadas de caprinos Criollos. $21^{\circ}$ Congreso Argentino y $2^{\circ}$ Congreso Uru- en corderos Santa Inés y por Vera et al. (2002 b) en caprinos Criollos, entre otros.

Se concluye que la evolución del PC y de la CE (altamente correlacionadas) de caprinos Criollos Serranos de un sistema pastoril subtropical, son influidos por la época de nacimiento.

guayo de Producción Animal. Paysandú. Uruguay. pp: 271-272.

Pérez Llano, B. and Mateos Rex, E. 1996. Effect of photoperiod on semen production and quality in bucks of Verata and Malaguena breeds. Small Ruminant Res., 22: 163-168.

Pinheiro, J.C. and Bates, D.M. 2000. Mixed-effects models in S and S-plus. Springer. New York. 512 pp.

Rabasa, A., Fernández, J. y Saldaño, S. 2001. Parámetros reproductivos de una majada caprina con manejo tradicional en el Dpto. Río Hondo (Santiago del Estero, Argentina). Zootecnia Trop., 19: 81-87.

R Development Core Team. 2006. R: A language and environment for statistical computing. R Foundation for Statistical Computing. Vienna. Austria. http://www.R-project.org (15/08/06). Souza, C.E., Moura, A.A., Lima, A.C. e Ciriaco, A.L. 2000. Desenvolvimento testicular, idade a puberdade e caracteristicas seminais em carneiros da raça Santa Inés no estado do Ceará. www.bovinos.ufc.br/sbs2000.pdf (12/ 04/06).

Vera, T., Chagra Dib, P. y Leguiza, D. 2002a. Influencia de la época del año sobre los valores de circunferencia escrotal de machos caprinos Criollos biotipo regional de los llanos de La Rioja. www.inta.gov.ar/larioja/info/documentos/ganadería/caprinos/Art_cap11.htm. 2 pp. (19/11/ 05).

Vera, T., Chagra Dib, P. y Leguiza, D. 2002b. Evolución de la circunferencia escrotal en caprinos Criollos biotipo regional, en los llanos de La Rioja. http://www.inta.gov.ar/larioja/info/documentos/ganadería/caprinos/Art_cap10htm. 2 pp. $(19 / 11 / 05)$.

Archivos de zootecnia vol. 59, núm. 226, p. 294. 